\title{
Density Functional Theory and Molecular Dynamics Simulations of Nanoporous Graphene Membranes for Hydrogen Separation
}

\author{
Supporting Information
}

\author{
Liliang Tian ${ }^{\mathrm{a}}$, Humin Duan ${ }^{\mathrm{a}}$, Jiaming Luo ${ }^{\mathrm{a}}$, Yonghong Cheng ${ }^{\mathrm{a}}$, Le Shi ${ }^{\mathrm{a}, *}$ \\ ${ }^{a}$ State key Laboratory of Electrical Insulation and Power Equipment, Centre of Nanomaterials for Renewable Energy, School \\ of Electrical Engineering, Xi'an Jiaotong University, Xi'an 710049, China.
}

\section{Construction of Doped NPG Models and NPG Models with Typical Defects}

After obtaining the structure information of the original NPG by DFT calculation, we designed two kinds of doped NPG model by replacing a C atom with a B or N atom in the centre of the NPG surface region. A single typical Stone-Wales defect (SW) structure in pristine graphene was introduced in the $1 \times 3$ NPG supercell (SW-NPG) to mimic the local defect in NPG. NPG doped with N atoms (N-NPG), NPG doped with B atoms (B-NPG) and SW-NPG were optimized by DFT calculation before constructing the MD gas separation simulation system.

\section{Potential Used by Gas Molecules}

The bond stretching in gas molecules is modelled by the harmonic potential $\left(\mathrm{CO}_{2}, \mathrm{H}_{2}, \mathrm{CH}_{4}\right)$, morse potential $\left(\mathrm{O}_{2}\right)$ and COMPASS $(\mathrm{CO})$ potential. The bond angle deformation is modelled by the harmonic potential in all the cases. The harmonic bond stretch potential is given by:

$$
E_{\text {bond }}=K_{\text {bond }}\left(r-r_{0}\right)^{2}
$$

where $K_{\text {bond }}$ is the bond coefficient and $r_{0}$ is the equilibrium bond distance. The harmonic angle deformation potential is given by:

$$
E_{\text {angle }}=K_{\text {angle }}\left(\theta-\theta_{0}\right)^{2}
$$

where $K_{\text {angle }}$ is the angle coefficient and $\theta_{0}$ is the equilibrium value of angle. The morse bond stretch potential is given by:

$$
E_{\text {bond }}=D\left(1-e^{-\alpha\left(r-r_{0}\right)^{2}}\right)
$$

where $r_{0}$ is the equilibrium bond distance, $\alpha$ is a stiffness parameter, and $D$ determines the depth of the potential well. The COMPASS bond stretch potential is given by:

$$
E_{\text {bond }}=K_{2}\left(r-r_{0}\right)^{2}+K_{3}\left(r-r_{0}\right)^{3}+K_{4}\left(r-r_{0}\right)^{4}
$$

where $K_{2}, K_{3}$ and $K_{4}$ are the bond coefficient and $r_{0}$ is the equilibrium bond distance. The parameters for the bond and angle harmonic potentials are listed in Table $\mathrm{S} 1$.

\footnotetext{
${ }^{*}$ Corresponding author

Email address: le.shi@mail.xjtu.edu.cn (Le Shi)
} 
Table S1: Bond and angle potential parameters used in the simulation.

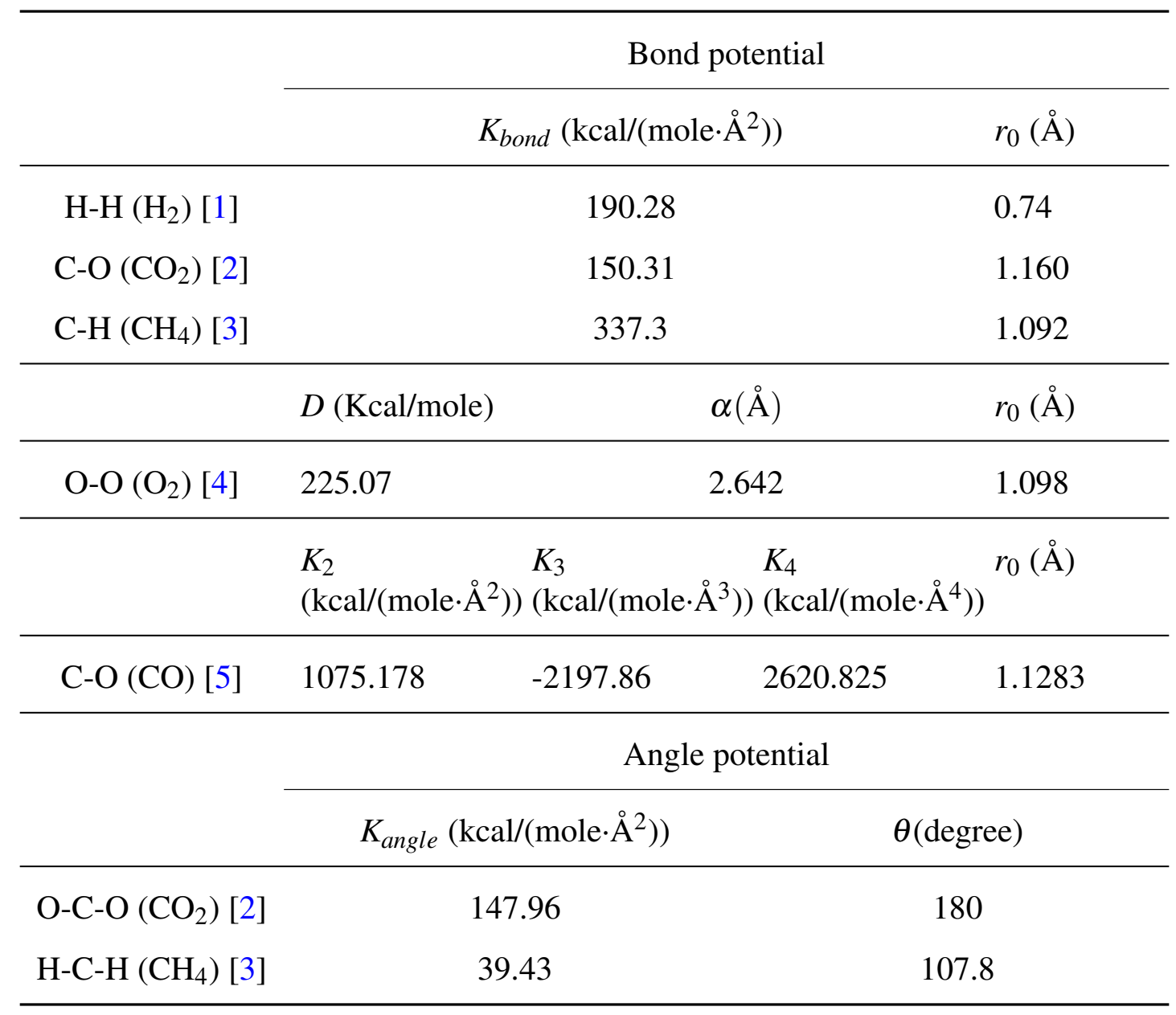




\section{Lennard-Jones and Coulombic Potential}

The Lennard-Jones (LJ) potentials and the Coulombic potentials are coupled together given by:

$$
\left.E=4 \varepsilon\left[\left(\frac{\sigma}{r}\right)^{12}-\left(\frac{\sigma}{r}\right)^{6}\right)\right]+\frac{C q_{i} q_{j}}{\gamma r} \quad\left(r<r_{c u t}\right)
$$

where $\varepsilon$ is energy scale, $\sigma$ is length scale, $C$ is an energy-conversion constant, $q_{i}$ and $q_{j}$ are the charges on the 2 atoms, and $\gamma$ is the dielectric constant which can be set by the dielectric command. In our simulations, the relative dielectric constants for all the gases were set as 1 . The Lennard-Jones and the Coulombic potential were set to $10 \AA$ for all interatomic interactions. Interaction parameters between cross atoms were obtained using the Lorentz-Berthelot mixing rule:

$$
\sigma_{s l}=\frac{\sigma_{s s}+\sigma_{l l}}{2}, \quad \varepsilon_{s l}=\sqrt{\varepsilon_{s s} * \varepsilon_{l l}}
$$

The parameters in Eq. (S5) for $\mathrm{H}_{2}, \mathrm{O}_{2}, \mathrm{CO}_{2}, \mathrm{CO}, \mathrm{CH}_{4}$ and NPG are listed in Table S2.

\begin{tabular}{|c|c|c|c|c|}
\hline & & $\varepsilon(\mathrm{kcal} / \mathrm{mole})$ & $\sigma(\AA)$ & charge \\
\hline $\mathrm{H}_{2}[1]$ & $\mathrm{H}-\mathrm{H}$ & 0.068 & 2.96 & 0 \\
\hline $\mathrm{O}_{2}[4]$ & $\mathrm{O}-\mathrm{O}$ & 0.2246 & 3.43 & 0 \\
\hline \multirow[t]{3}{*}{$\mathrm{CO}_{2}[2]$} & $\mathrm{C}-\mathrm{C}$ & 0.056 & 2.757 & 0.6512 \\
\hline & $\mathrm{O}-\mathrm{O}$ & 0.160 & 3.033 & -0.3256 \\
\hline & $\mathrm{C}-\mathrm{O}$ & 0.095 & 2.895 & - \\
\hline \multirow[t]{3}{*}{$\mathrm{CO}$ [6] } & $\mathrm{C}-\mathrm{C}$ & 0.126 & 3.797 & 0.0203 \\
\hline & $\mathrm{O}-\mathrm{O}$ & 0.09 & 3.656 & -0.0203 \\
\hline & C-O & 0.105 & 0.4394 & - \\
\hline \multirow[t]{3}{*}{$\mathrm{CH}_{4}[7]$} & $\mathrm{C}-\mathrm{C}$ & 0.066 & 3.5 & -0.24 \\
\hline & $\mathrm{H}-\mathrm{H}$ & 0.03 & 2.5 & 0.06 \\
\hline & C-H & 0.045 & 3 & - \\
\hline \multirow[t]{4}{*}{ NPG } & C-C [2] & 0.056 & 3.4 & - \\
\hline & C-H [2] & 0.03 & 2.5 & - \\
\hline & B-B [8] & 0.095 & 3.453 & - \\
\hline & $\mathrm{N}-\mathrm{N}[8]$ & 0.145 & 3.365 & - \\
\hline
\end{tabular}

Table S2: Parameters in the potential model Eqtion S5 of gas molecules. 


\section{Electron Density Isosurfaces for Gases Permeating Through NPG}

The electron density diagrams of other gases permeating through the NPG are shown in Figure S1.

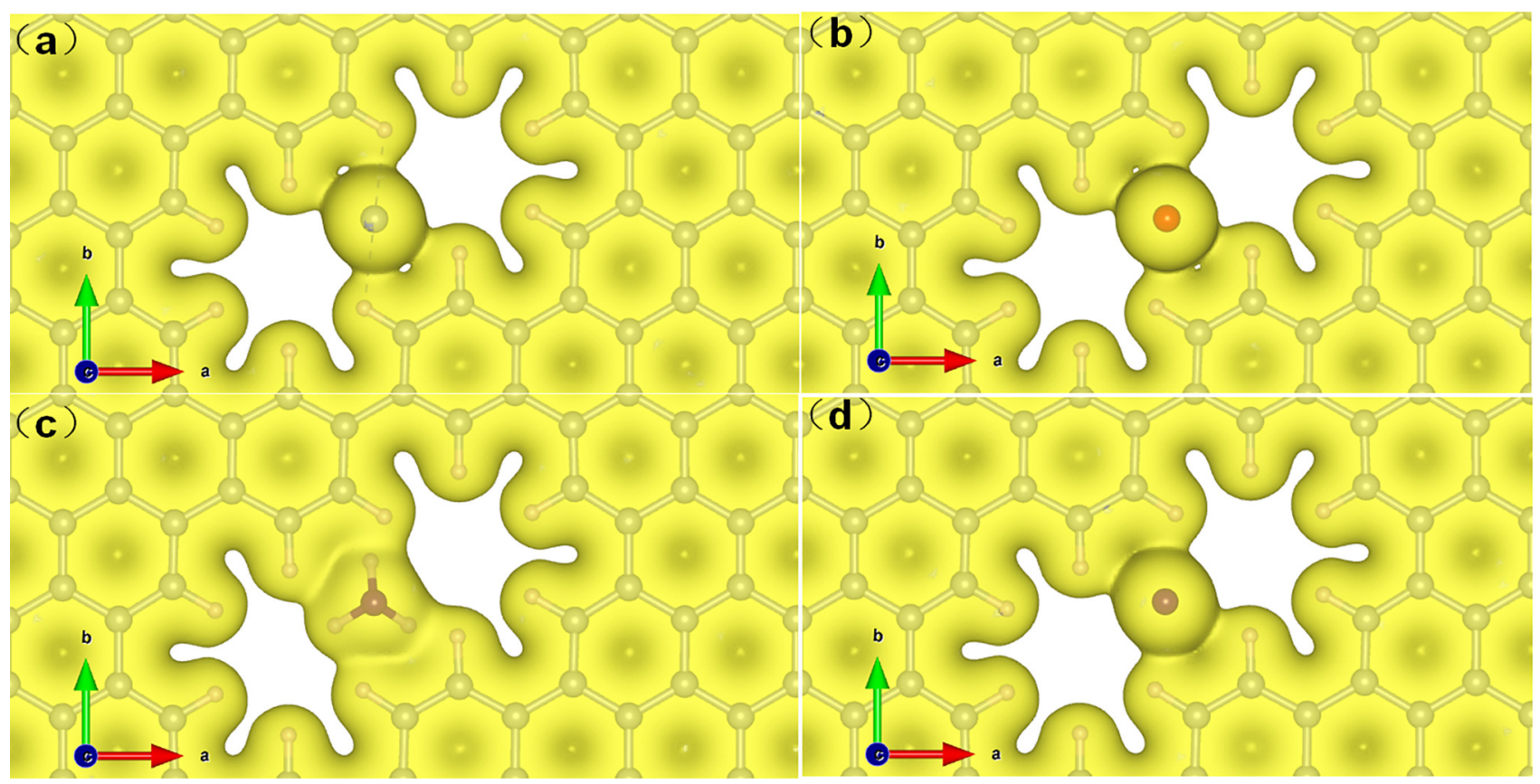

Figure S1: Electron density isosurfaces for (a) $\mathrm{CO}$, (b) $\mathrm{CO}_{2}$, (c) $\mathrm{NH}_{3}$ (d) $\mathrm{N}_{2}$ molecules passing through NPG. The value of isosurfaces is $0.02 \mathrm{e} / \AA^{3}$.

\section{Good Mechanical Stability of NPG}


(a)

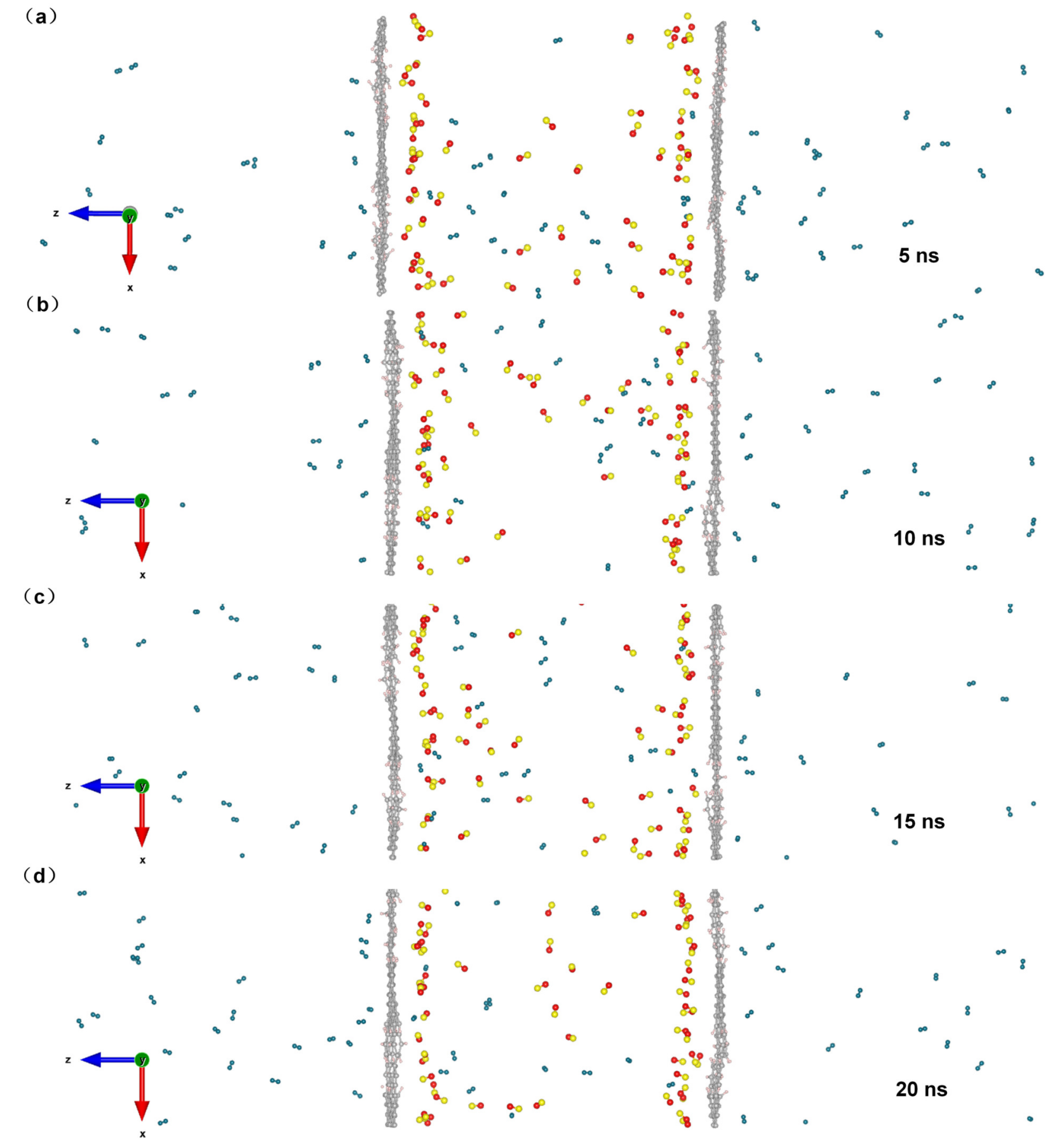

Figure S2: Snapshots of the simulated system of $\mathrm{H}_{2} / \mathrm{CO}$ combination at (a) $5 \mathrm{~ns}$ (b) $10 \mathrm{~ns}$ (c) $15 \mathrm{~ns}$ (d) $20 \mathrm{~ns}$. 


\section{Other Gas Separation MD Simulation Results}

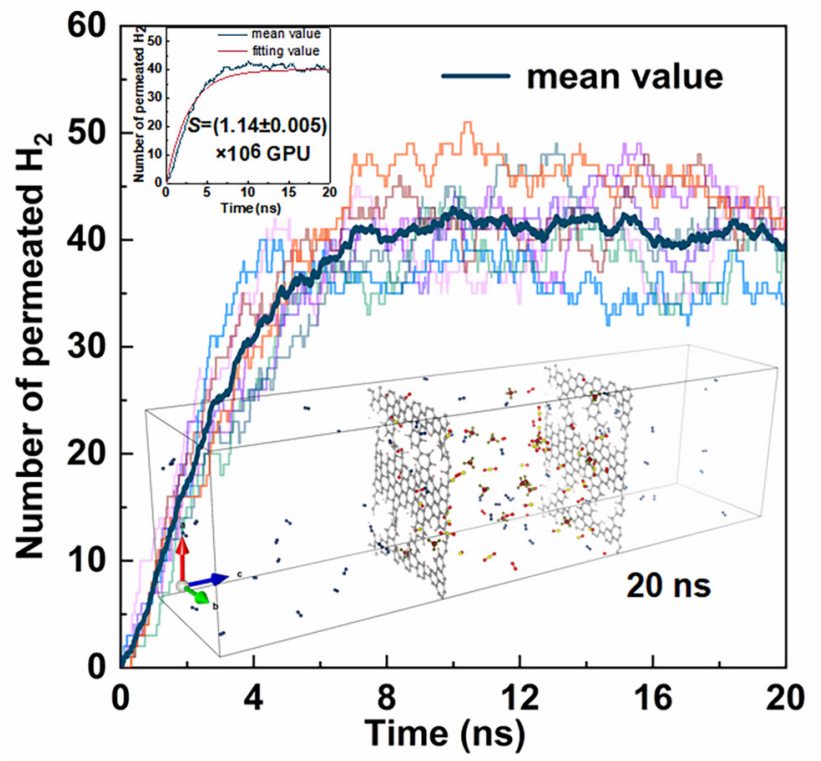

Figure S3: Molecular dynamics simulations of $\mathrm{H}_{2}$ permeation results for combination of $\mathrm{H}_{2} / \mathrm{CO}_{2} / \mathrm{CO} / \mathrm{O}_{2} / \mathrm{CH}_{4}$. The slim rainbow coloured line is the result of ten single runs, the thick brown line is the average of the results of all runs. The insets in the upper left corner show the average values and their corresponding fitted values and the $\mathrm{S}$ in the inset is the permeability of $\mathrm{H}_{2}$ converted from the fitted results. The insets on the bottom side are snapshots of the simulated system at $20 \mathrm{~ns}$. 

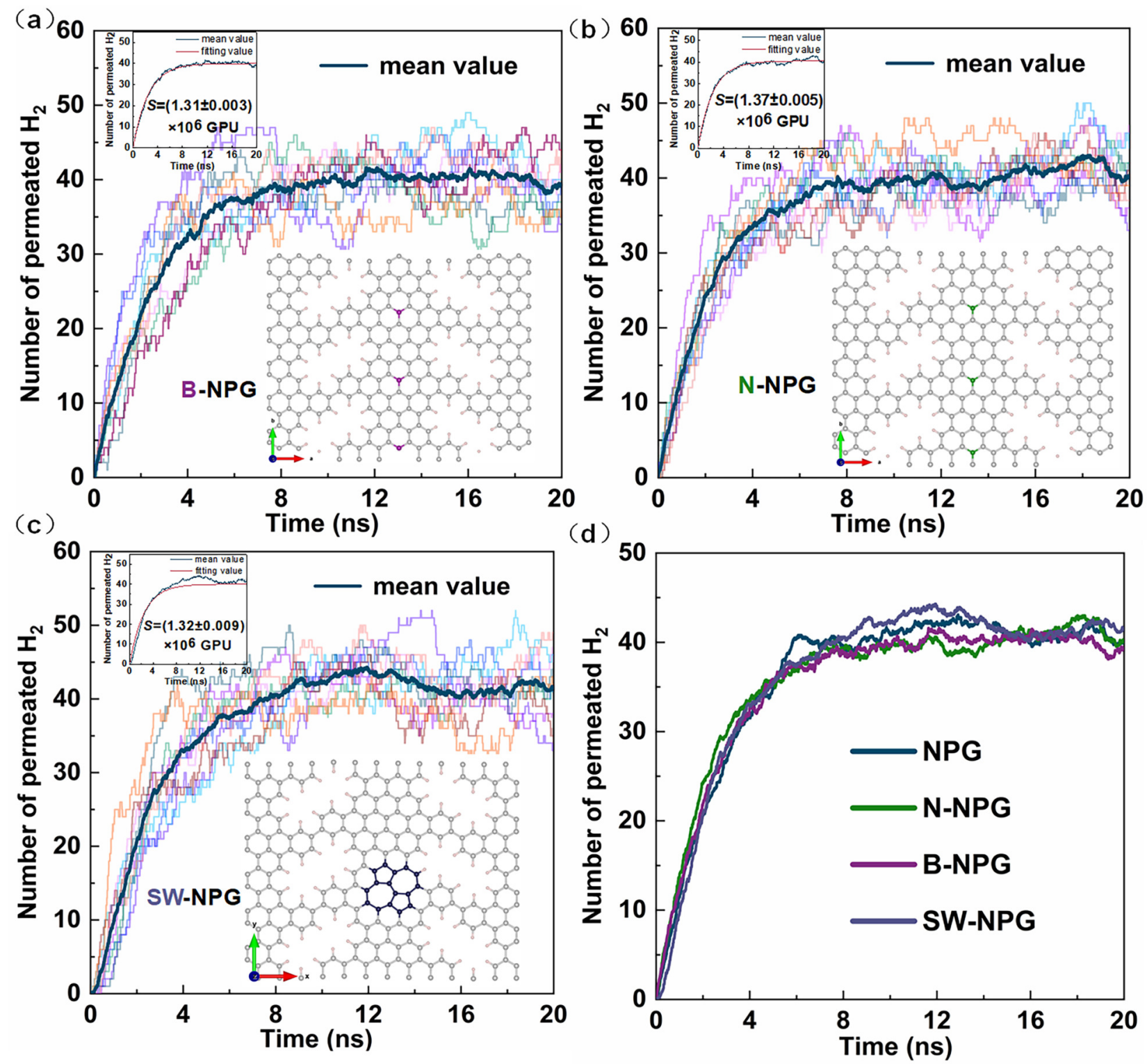

Figure S4: Molecular dynamics simulations of $\mathrm{H}_{2}$ permeation results for $\mathrm{H}_{2} / \mathrm{CO}$ combination in (a) B-NPG (b) N-NPG (c) SW-NPG gas separation simulation system. The slim rainbow coloured line is the result of ten single runs, the thick brown line is the average of the results of all runs. The insets in the upper left corner show the average values and their corresponding fitted values and the $\mathrm{S}$ in the inset is the permeability of $\mathrm{H}_{2}$ converted from the fitted results. The insets on the bottom side are snapshots of the simulated system at $20 \mathrm{~ns}$. (d) Comparison of mean values for MD simulation results of $\mathrm{H}_{2} / \mathrm{CO}$ combination in NPG, N-NPG, B-NPG and SW-NPG gas separation simulation systems. 


\section{Effect of Rigid NPG on $\mathrm{H}_{2}$ Permeability}

Many previous work used rigid NPG models in their simulations, but no article has discussed the effect of rigid NPG models on the accuracy of calculated gas permeability. For this reason, we explored the permeation behaviour of $\mathrm{H}_{2}$ using a rigid NPG model and compared the results with the flexible NPG model under the same conditions. Taking the results of $\mathrm{H}_{2} / \mathrm{O}_{2}$ gas mixture simulation as an example, the rigid NPG destroys the adsorption properties of $\mathrm{O}_{2}$ on its surface, as shown in Figure S5. Compared with the flexible NPG, the molecular number share of $\mathrm{O}_{2}$ in the adsorption layer plummets to $50 \%$ in the first 2 ns. Correspondingly, the number of $\mathrm{H}_{2}$ adsorbed increases, and thus the permeability of $\mathrm{H}_{2}$ permeability increases. Fitting the average value of ten simulations from 2 ns onwards, the permeability of $\mathrm{H}_{2}$ obtained is increased by $51 \%$ compared to that of the flexible NPG as shown in Figure S6. Considering that the 2D membrane fluctuation at nanoscale cannot be restrained in real applications, we believe that the flexible NPG model is more appropriate for the calculation of permeability in the mixed gas model. .

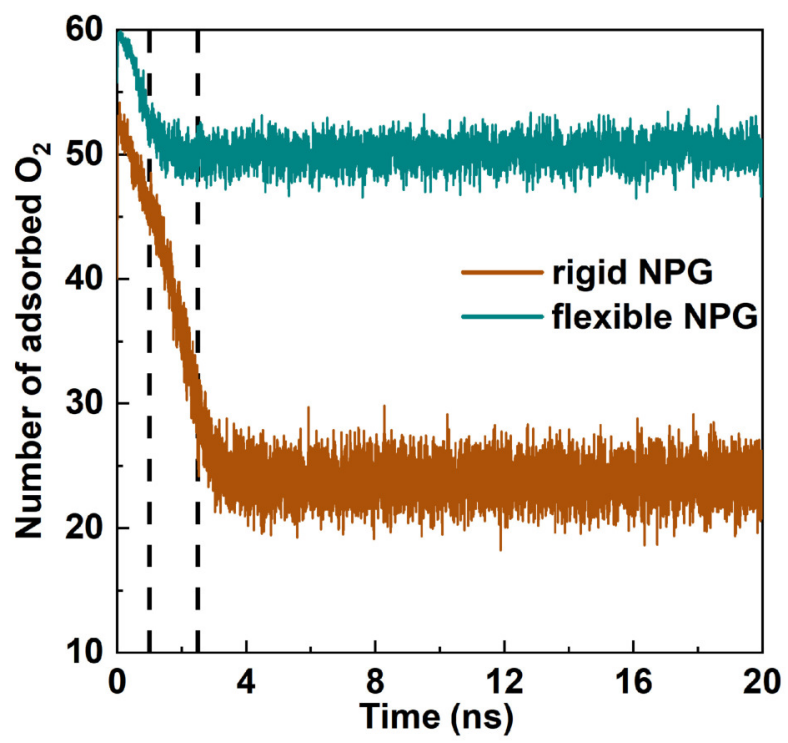

Figure S5: Variation of the amount of $\mathrm{O}_{2}$ in the adsorption layer of $\mathrm{H}_{2} / \mathrm{O}_{2}$ gas mixture with simulation time under the rigid NPG and flexible NPG models. 

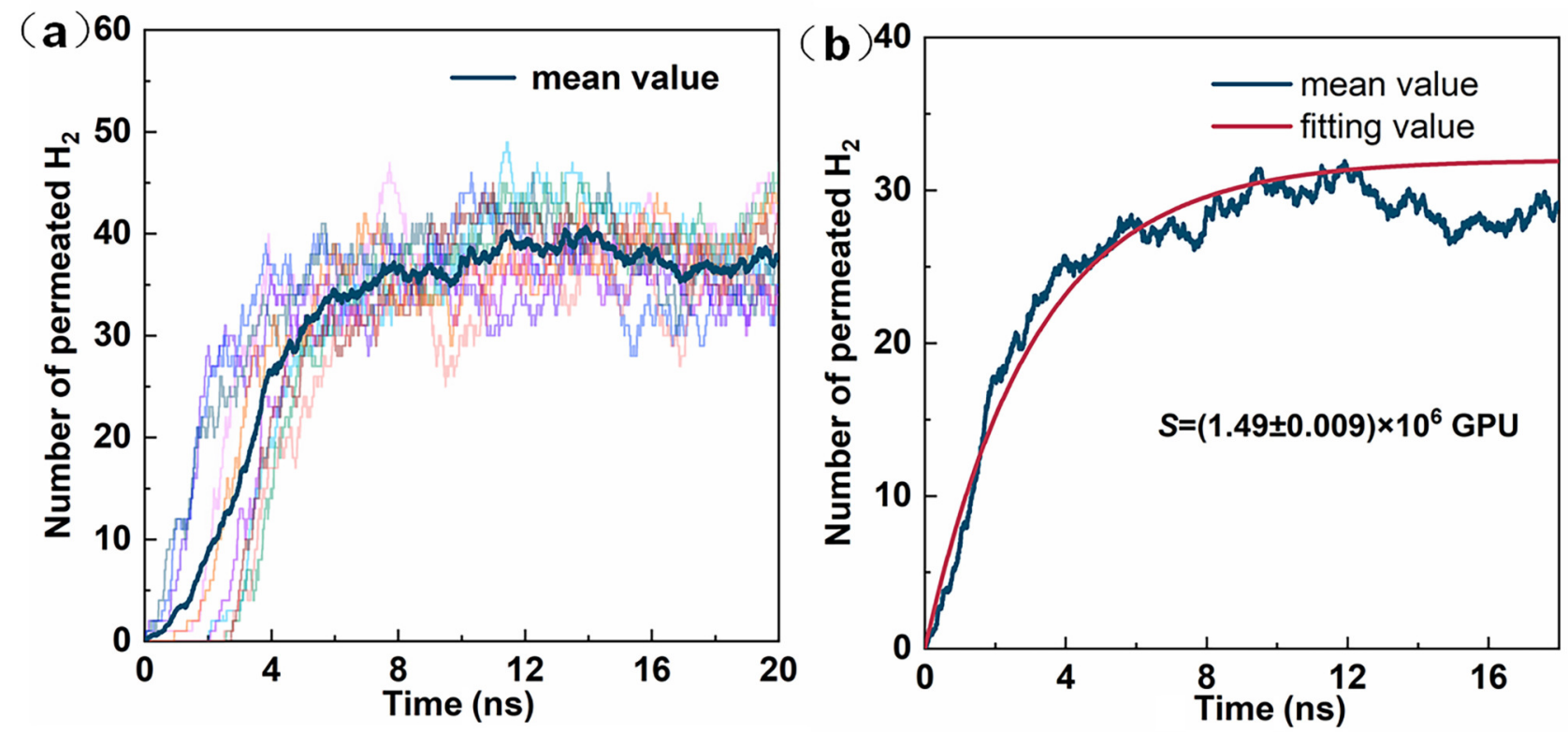

Figure S6: (a) Number and average of $\mathrm{H}_{2}$ permeation for ten simulations in $\mathrm{H}_{2} / \mathrm{O}_{2}$ mixture using rigid NPG model. (b) Fitting of the mean value after $2 \mathrm{~ns}$

\section{References}

[1] M. Nouri, K. Ghasemzadeh, A. Iulianelli, Theoretical Evaluation of Graphene Membrane Performance for Hydrogen Separation Using Molecular Dynamic Simulation, Membranes 9 (2019) 110. URL: https : //www .mdpi .com/2077-0375/9/9/110. doi:10.3390/membranes9090110.

[2] C. Sun, B. Wen, B. Bai, Application of nanoporous graphene membranes in natural gas processing: Molecular simulations of $\mathrm{CH} 4 / \mathrm{CO} 2, \mathrm{CH} 4 / \mathrm{H} 2 \mathrm{~S}$ and $\mathrm{CH} 4 / \mathrm{N} 2$ separation, Chemical Engineering Science 138 (2015) 616-621. URL: http://www.sciencedirect.com/science/article/pii/ S0009250915006156. doi:10.1016/j.ces.2015.08.049.

[3] X. Zhao, H. Jin, Y. Chen, Z. Ge, Numerical study of H2 , CH4, CO, O2 and CO2 diffusion in water near the critical point with molecular dynamics simulation, Computers \& Mathematics with Applications 81 (2021) 759-771. URL: https://linkinghub.elsevier.com/retrieve/ pii/S0898122119305450. doi:10.1016/j. camwa.2019.11.012.

[4] M. Darvishi, M. Foroutan, Mechanism of water separation from a gaseous mixture via nanoporous graphene using molecular dynamics simulation, RSC Advances 5 (2015) 81282-81294. URL: http: //xlink.rsc.org/?DOI=C5RA16452D. doi:10.1039/C5RA16452D.

[5] J. P. Bouanich, Site-site Lennard-Jones potential parameters for N2, O2, H2, CO and CO2, Journal of Quantitative Spectroscopy and Radiative Transfer 47 (1992) 243-250. URL: https://www. sciencedirect.com/science/article/pii/002240739290142Q. doi:10. 1016/0022-4073(92) 90142-Q.

[6] J. Wilson, N. Faginas-Lago, J. Vekeman, I. G. Cuesta, J. Sánchez-Marín, A. Sánchez de Merás, Modeling the Interaction of Carbon Monoxide with Flexible Graphene: From Coupled Cluster Calculations to Molecular-Dynamics Simulations, ChemPhysChem 19 (2018) 
774-783. URL: https://chemistry-europe.onlinelibrary.wiley.com/doi/abs/10.1002/ cphc. 201701387. doi:10.1002/cphc. 201701387.

[7] H. Liu, Z. Chen, S. Dai, D.-e. Jiang, Selectivity trend of gas separation through nanoporous graphene, Journal of Solid State Chemistry 224 (2015) 2-6. URL: http: //www . sciencedirect . com/science/article/pii/S0022459614000346. doi:10.1016/j.jssc.2014.01.030.

[8] A. Hassani, M. T. H. Mosavian, A. Ahmadpour, N. Farhadian, Improvement of methane storage in nitrogen, boron and lithium doped pillared graphene: A hybrid molecular simulation, Journal of Natural Gas Science and Engineering 46 (2017) 265-274. URL: https: //www . sciencedirect. com/science/article/pii/S1875510017303189. doi:10.1016/j.jngse.2017.07.021. 